\title{
Pion reactions with few-nucleon systems
}

\author{
Vadim Baru*, \\ Forschungszentrum Jülich, Institut für Kernphysik (Theorie) and Jülich Center for Hadron \\ Physics, D-52425 Jülich, Germany and \\ Institute for Theoretical and Experimental Physics, B. Cheremushkinskaya 25, \\ 117218 Moscow, Russia \\ E-mail: v.baruefz-juelich.de
}

\begin{abstract}
We report about the recent results for s- and p-wave pion production in $N N \rightarrow N N \pi$ within effective field theory and discuss how the charge symmetry breaking in $p n \rightarrow d \pi^{0}$ can be used to extract the strong contribution to the neutron-proton mass difference.
\end{abstract}

6th International Workshop on Chiral Dynamics, CD09

July 6-10, 2009

Bern, Switzerland

${ }^{*}$ Speaker. 


\section{Introduction}

With the advent of chiral perturbation theory (ChPT), the low-energy effective field theory (EFT) of QCD, high accuracy calculations for hadronic reactions with a controlled error estimation have become possible [1,2]. The framework has been successfully applied to study, in particular, $\pi \pi$ [3] and $\pi N$ [4] scattering observables as well as nuclear forces [5]. In this contribution we discuss an application of $\mathrm{ChPT}$ to the reactions involving pion production on two-nucleon systems. This type of reactions allows one to test predictions of ChPT in the process with the large momentum transfer typical for the production process. As was first advocated in Refs. [6,7], the initial nucleon momentum in the threshold kinematics sets the new "small" scale in the problem, namely, $p \simeq \sqrt{m_{N} M_{\pi}} \simeq 360 \mathrm{MeV}\left(\chi \simeq p / m_{N} \simeq \sqrt{M_{\pi} / m_{N}}\right)$, where $M_{\pi}\left(m_{N}\right)$ is the pion (nucleon) mass. The proper way to include this scale in the power counting was presented in Ref. [8] and implemented in Ref. [9], see Ref. [10] for a review article. As a consequence, the hierarchy of diagrams changes in the modified power counting scheme of Ref. [8], and some loops start to contribute already at NLO for $s$-wave pion production. On the other hand, $p$-wave pion production is governed by the tree-level diagrams up to NNLO. In what follows we discuss the status of the theory for pion production in the isospin conserving and isospin violating case. It was first argued in Ref. [11] that charge symmetry breaking (CSB) effects recently observed experimentally in $p n \rightarrow d \pi^{0}$ [12] provide an access to the neutron-proton mass difference which is the fundamental quantity of QCD. To take this challenge, however, one needs to have the isospin conserving case fully under control. In sec. 2 we briefly discuss the theoretical status for s-wave pion production. Sec. 3 is devoted to a more detailed discussion of the recent results for p-wave pion production. In sec. 4 we briefly highlight the recent developments in the study of charge symmetry breaking effects in $p n \rightarrow d \pi^{0}$. We finalize with the summary of the latest results.

\section{2. s-wave pion production and the concept of reducibility}

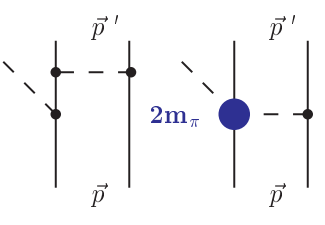

LO

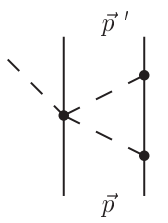

b

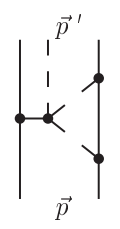

C

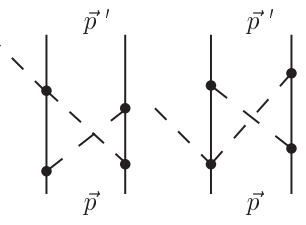

d1 $\mathrm{d} 2$

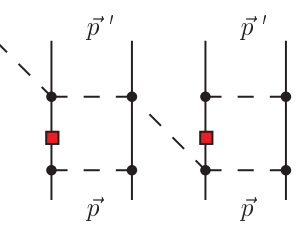

a1

NLO

Figure 1: Complete set of nucleonic diagrams up to NLO. Note that sum of all loops at NLO vanishes.

A method how to calculate processes on few nucleon systems with external probes was proposed by Weinberg [13]:

1. the perturbative transition (production) operators have to be calculated systematically using ChPT. They should consist of irreducible graphs only.

2. the transition operators have to be convoluted with the non-perturbative $N N$ wave functions. 


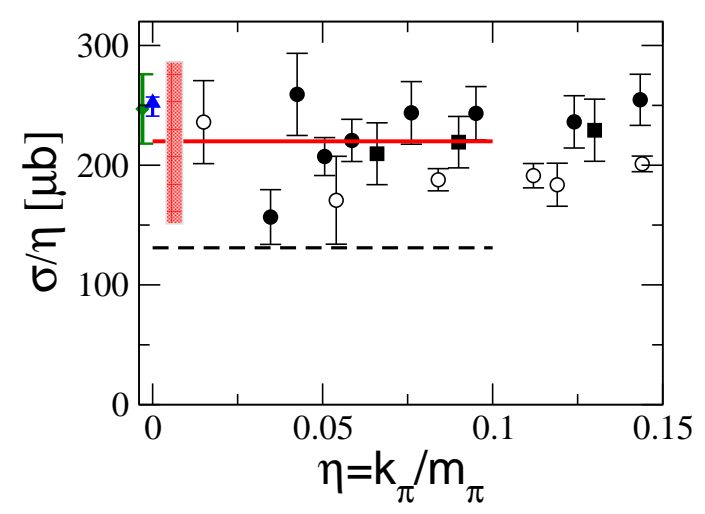

Figure 2: Comparison of our results to experimental data for $N N \rightarrow d \pi$. The data sets are from Refs. [17-21]. Note that the green diamond and the blue triangle correspond to the most resent measurements from pionic deuterium atom $[20,21]$ at $\eta=0$. The dashed line corresponds to the model of Koltun and Reitan [22]. The solid red curve represents our results, as given in Ref. [14], the filled red box demonstrates the theoretical uncertainty of the NLO calculation.

Therefore it is necessary to disentangle those diagrams that are part of the wave function from those that are part of the transition operator. In complete analogy to $N N$ scattering, the former are called reducible and the latter irreducible. The distinction stems from whether the diagram shows a twonucleon cut or not. Thus, in accordance to this rule, the one-loop diagrams shown in Fig. 1(b)-(d) are irreducible, whereas diagrams (a) seem to be reducible. This logic was used in Ref. [9] to single out the irreducible loops contributing at NLO. The findings of Ref. [9] were:

- For the channel $p p \rightarrow p p \pi^{0}$ the sum of diagrams (b)-(d) of Fig. 1 vanished due to a cancellation between individual diagrams

- For the channel $p p \rightarrow d \pi^{+}$the same sum gave a finite answer ${ }^{1}$ :

$$
A_{p p \rightarrow d \pi^{+}}^{b+c+d}=\frac{g_{A}^{3}}{256 f_{\pi}^{5}}(-2+3+0)|\vec{q}|=\frac{g_{A}^{3}|\vec{q}|}{256 f_{\pi}^{5}}
$$

where $f_{\pi}$ denotes the pion decay constant and $g_{A}$ is the axial-vector coupling of the nucleon.

The latter amplitude grows linearly with increasing final $N N$-relative momentum $|\vec{q}|$, which leads to a large sensitivity to the final $N N$ wave function, once the convolution of those with the transition operators is evaluated. However, the problem is that such a sensitivity is not allowed in a consistent field theory as was stated in Ref. [15]. The solution of this problem was presented in Ref. [14] (see also Ref. [16]). It was pointed out in Ref. [14] that the diagrams that look formally reducible can acquire irreducible contributions in the presence of the energy-dependent vertices (or timedependent Lagrangian densities). Specifically, the energy dependent part of the leading WeinbergTomozawa (WT) $\pi N \rightarrow \pi N$ vertex cancels one of the intermediate nucleon propagators (see the one with the red square in Fig. 1), resulting in an additional irreducible contribution at NLO. It turned out that this additional irreducible contribution compensates the linear growth of diagrams (b)-(d) thus solving the problem. Thus, up to NLO, only the diagrams appearing at LO (see Fig. 1), contribute to $p p \rightarrow d \pi^{+}$, with the rule that the $\pi N \rightarrow \pi N$ vertex is put on-shell. The latter stems from the observation that in addition to the leading WT-term $\left(\sim 3 M_{\pi} / 2\right)$ the nucleon recoil correction to the WT $\pi N$ vertex $\left(\sim M_{\pi} / 2\right)$ also contributes at LO. As a result the dominant $\pi N$ rescattering amplitude is enhanced by a factor of $4 / 3$ as compared to the traditionally used value $\left(\sim 3 M_{\pi} / 2\right)$, which leads to a good description of the experimental data for $p p \rightarrow d \pi^{+}$(see Fig. 2).

\footnotetext{
${ }^{1}$ The connection of the amplitude $A$ to the observables is given, e.g., in Ref [14]
} 


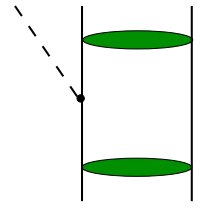

$\mathrm{LO}$

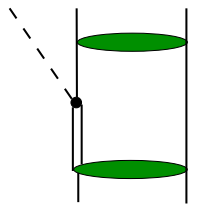

NLO

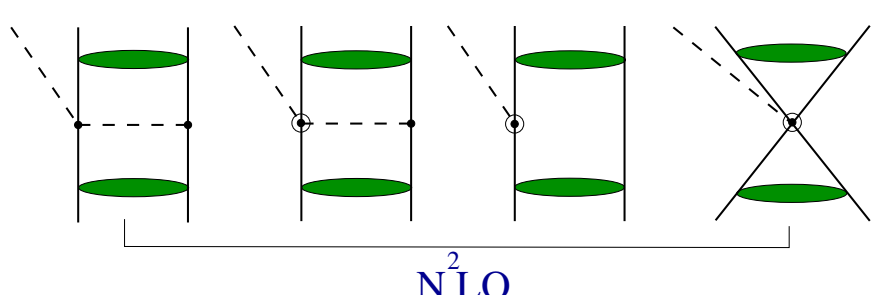

$\mathrm{N}^{2} \mathrm{LO}$

Figure 3: Diagrams that contribute to the $p$-wave amplitudes of $N N \rightarrow N N \pi$ up to NNLO.
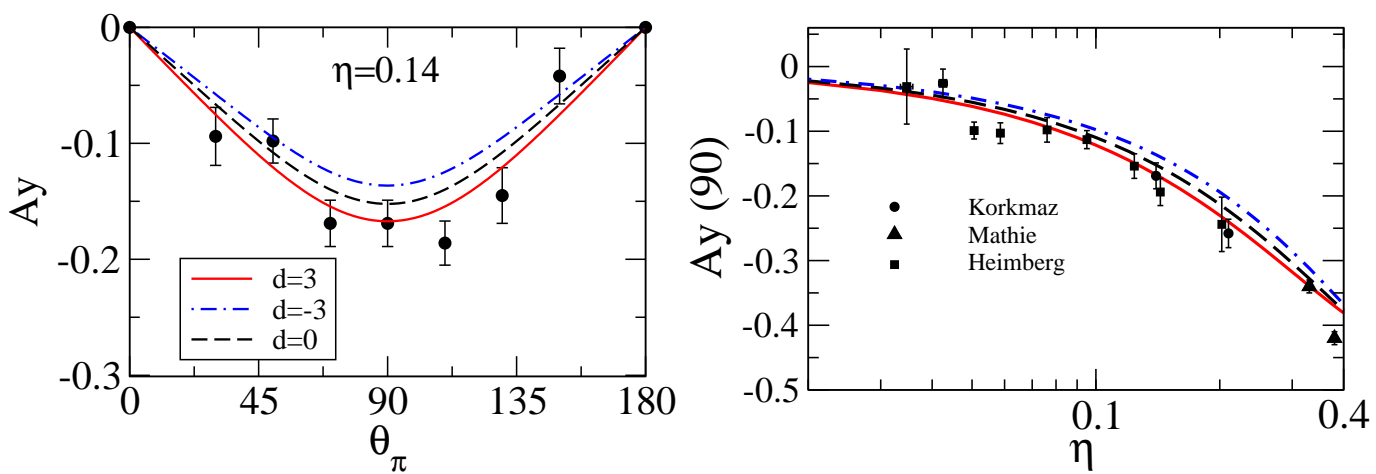

Figure 4: Results for the analyzing power at $\eta=0.14$ (left panel) and the analyzing power at 90 degrees (right panel) for the reaction $p p \rightarrow d \pi^{+}$for different values of the LEC $d$ (in units $1 /\left(f_{\pi}^{2} M_{N}\right)$ ) of the $(\bar{N} N)^{2} \pi$ contact operator. Shown are $d=3$ (red solid line), $d=0$ (black dashed line), and $d=-3$ (blue dot-dashed line). The data are from Refs. [33-37].

Note, however, that the relatively large theoretical uncertainty of about $2 M_{\pi} / m_{N} \approx 30 \%$ for the cross section requires a carefull study of higher order effects.

\section{3. p-wave pion production}

Diagrams that contribute to $\mathrm{p}$-wave pion production up to NNLO in the modified power counting are shown in Fig.3. In particular, at NNLO there are subleading rescattering and direct pion production operators as well as the $(\bar{N} N)^{2} \pi$ contact term. Notice that it is the same contact term that also contributes to the three-nucleon force [8,23], to the processes $\gamma d \rightarrow \pi N N[24,25]$ and $\pi d \rightarrow \gamma N N[26,27]$ as well as to weak reactions such as, e.g., tritium beta decay and proton-proton $(p p)$ fusion $[28,29]$. Therefore it provides an important connection between different low-energy reactions. It is getting even more intriguing once one realizes that this operator appears in the above reactions in very different kinematics, ranging from very low energies for both incoming and outgoing $N N$ pairs in $p d$ scattering and the weak reactions up to relatively high initial energies for the $N N$ induced pion production. As a part of this connection in Ref. [30] it was shown that both the ${ }^{3} \mathrm{H}$ and ${ }^{3} \mathrm{He}$ binding energies and the triton $\beta$-decay can be described with the same contact term. However, an apparent discrepancy between the strength of the contact term needed in $p p \rightarrow p n \pi^{+}$ and in $p p \rightarrow d e^{+} v_{e}$ was reported in Ref. [31]. If the latter observation were true, it would question the applicability of chiral EFT to the reactions $N N \rightarrow N N \pi$. To better understand the discrepancy reported in Ref. [31], in the recent paper [32] we simultaneously analyzed different pion produc- 

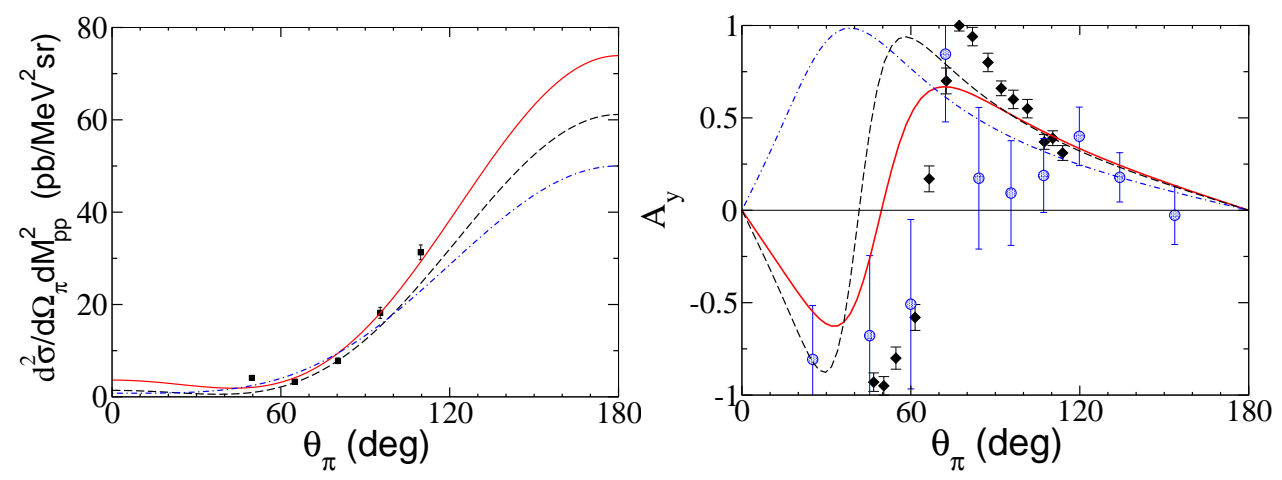

Figure 5: Results for $d^{2} \sigma / d \Omega_{\pi} d M_{p p}^{2}$ (left panel) and $A_{y}$ (right panel) for $p n \rightarrow p p\left({ }^{1} S_{0}\right) \pi^{-}$. Shown are the results for $d=3$ (red solid line), $d=0$ (black dashed line) and $d=-3$ (blue dot-dashed line). The data are from TRIUMF [38, 39] (black squares) and from PSI [40] (blue circles) .

tion channels. In particular, we calculated the $p$-wave amplitudes for the reactions $p n \rightarrow p p \pi^{-}$, $p p \rightarrow p n \pi^{+}$, and $p p \rightarrow d \pi^{+}$. Note that even in these channels the contact term occurs in entirely different dynamical regimes. For the first channel $p$-wave pion is produced along with the slowly moving protons in the ${ }^{1} S_{0}$ final state whereas for the other two channels the ${ }^{1} S_{0} p p$ state is to be evaluated at the relatively large initial momentum. In practice, we varied the value of the lowenergy constant (LEC) $d$, which represents the strength of the contact operator, in such a way to get the best simultaneous qualitative description of all channels of $N N \rightarrow N N \pi$. It should be stressed, however, that the value of $d$ depends on the $N N$ interaction employed and on the method used to regularize the overlap integrals. It therefore does not make much sense to compare values for $d$ as found in different calculations. Instead one should compare results on the level of observables and this is what we do below (see also Ref. [32]). In Fig.4 we compare our results for various values of $d$ with the experimental data for the analyzing power for the reaction $p p \rightarrow d \pi^{+}$. We find that the data prefer a positive value of $d$ of about 3. A similar pattern can be observed in the reaction $p n \rightarrow p p \pi^{-}$as illustrated in Fig. 5. Again the data show a clear preference of the positive value for LEC $d$ - our fit resulted in $d=3$ for the best value. This channel, however, has been measured at TRIUMF at relatively large excess energy $(\eta=0.66)$ where the conclusion may be spoiled due to the onset of pion d-waves. Fortunately, a new measurement for the same observables at lower energies is currently ongoing at COSY [41] which will soon allow a quantitative extraction of the value of the LEC $d$. We now turn to the reaction $p p \rightarrow p n \pi^{+}-$this channel was used in the analysis of Ref. [31]. The reaction $p p \rightarrow p n \pi^{+}$should have, in principle, the same information on the LEC $d$ as contained in the deuteron channel. However, it is much more difficult to extract the pertinent information unambiguously from this reaction. In particular, the final $\mathrm{NN}$-system might be not only in $S$ - but also in $P$-wave both for isospin-zero and for isospin-one NN states. At the energies considered in the experimental investigation, $\eta=0.22,0.42$, and 0.5 , the $P p$ amplitudes may contribute significantly [43-45]. In the current study these states are disregarded. The results of our calculation for the magnitude $A_{2}$ are given ${ }^{2}$ in the left panel of Fig. 6 . One finds again

\footnotetext{
${ }^{2}$ The coefficients $A_{i}$ are related to the unpolarized differential cross section via $\frac{d \sigma}{d \Omega}=A_{0}+A_{2} P_{2}(x)$, with $P_{2}(x)$ being the second Legendre polynomial
} 

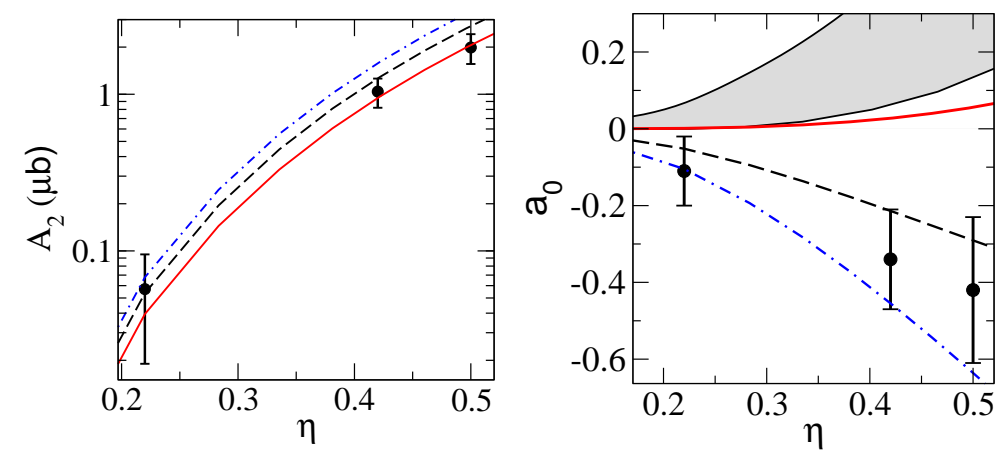

Figure 6: Results for the magnitude $A_{2}$ (left panel) and the partial wave amplitude $a_{0}(\sqrt{\mu b})$ representing the relevant transition ${ }^{1} S_{0} \rightarrow{ }^{3} S_{1} p$ (right panel) for $p p \rightarrow$ $p n \pi^{+}$for different values of the LEC $d$. The notation is the same as in Fig. 5, gray band corresponds to the results of Ref. [31]. The data are from Ref. [42].

that the positive LEC $d \simeq 3$ seems to be preferred. Thus we conclude that all reaction channels of $N N \rightarrow N N \pi$ can be described simultaneously with the same value of the LEC $d$. Coming back to the problem raised in Ref. [31] it should be pointed out that the results of this work were not compared directly to the observables in $p p \rightarrow p n \pi^{+}$. Instead, they were compared to the results of the partial wave analysis (PWA) performed in Ref. [42], as demonstrated in the right panel of Fig. 6. It is based on this discrepancy between data and theory it was concluded in Ref. [31] about the failure of simultaneous description of the weak processes and $N N \rightarrow N N \pi$. However, the partial wave analysis of Ref. [42] seems to be not correct. Here we refer the interested reader to Ref. [32] where the drawbacks of this PWA are discussed in detail. To illustrate the problems of the PWA in the right panel of Fig. 6 we also show the results of our calculation for the relevant partial wave $a_{0}$ which corresponds to the transition ${ }^{1} S_{0} \rightarrow{ }^{3} S_{1} p$ where the contact term acts. Clearly, although all data presented in Ref. [42] are in a good agreement with our calculation (see left panel in Fig. 6 and also Ref. [32] for more details), the partial wave amplitude $a_{0}$ is not at all described. Thus, we think that the problem with the simultaneous description of $p p \rightarrow d e^{+} v_{e}$ and $p p \rightarrow p n \pi^{+}$, raised in Ref. [31], is due to the drawbacks of the partial wave analysis of Ref. [42].

\section{CSB effects in $p n \rightarrow d \pi^{0}$}

Recently, experimental evidence for CSB was found in reactions involving the production of neutral pions. At IUCF non-zero values for the $d d \rightarrow \alpha \pi^{0}$ cross section were established [46]. At TRIUMF a forward-backward asymmetry of the differential cross section for $p n \rightarrow d \pi^{0}$ was reported which amounts to $A_{f b}=[17.2 \pm 8$ (stat.) \pm 5.5 (sys.) $] \times 10^{-4}$ [12]. In a charge symmetric world the initial $p n$ pair would consist of identical nucleons in a pure isospin one state. Thus the apparent forward-backward asymmetry is due to charge symmetry breaking.

The neutron-proton mass difference is due to strong and electromagnetic interactions [47], i.e. $\delta m_{N}=m_{n}-m_{p}=\delta m_{N}^{\mathrm{str}}+\delta m_{N}^{\mathrm{em}}$. It was stressed and exploited in Ref. [11] that the strength of the rescattering CSB operator at LO in $p n \rightarrow d \pi^{0}$ (see Fig. 4(a)) is proportional to a different combination of $\delta m_{N}^{\text {str }}$ and $\delta m_{N}^{\mathrm{em}}$ ( see also $[48,49]$ for related works). Thus the analysis of CSB effects in $p n \rightarrow d \pi^{0}$ should allow to determine these important quantities individually. It was, however, quite surprising to find that, using the values for $\delta m_{N}^{\mathrm{str}}$ and $\delta m_{N}^{\mathrm{em}}$ from Ref. [47], the leading order calculation of the forward-backward asymmetry [11] over-predicted the experimental value by about a factor of 3 - a consistent description would call for an agreement with data within the theoretical uncertainty of $15 \%$ for this kind of calculation. The evaluation of certain higher 


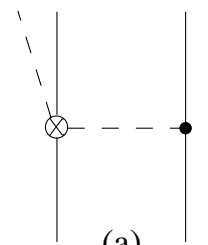

(a)

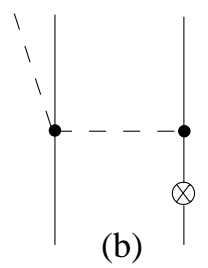

(b)
Figure 7: Leading order diagrams for the isospin violating $s$ wave amplitudes of $p n \rightarrow d \pi^{0}$. Diagram (a) corresponds to isospin violation in the $\pi N$ scattering vertex explicitly whereas diagram (b) indicates an isospin-violating contribution due to the neutron-proton mass difference in conjunction with the timedependent Weinberg-Tomozawa operator.

order corrections performed in Ref. [11] and in a recent study [50] did not change the situation sufficiently — the significant overestimation of the data persisted. In the recent work [51] we have shown that there is one more rescattering operator that contributes at LO (see diagram (b) in Fig. 4). In full analogy to isospin conserving s-wave pion production, the idea was based on the fact that the energy-dependent WT $\pi N$ vertex acquires an additional contribution proportional to $\delta m_{N}$ as soon as we distinguish between the proton and the neutron. We evaluated this new LO operator and recalculated the forward-backward assymetry at LO. It should be pointed out at this stage that $A_{f b}$ at $\mathrm{LO}$ is proportional to the interference of the s-wave pion CSB amplitude at LO and the p-wave pion isospin conserving amplitude. The latter is calculated up to NNLO, as discussed in Sec.3, and exhibits very good description of data, which is a necessary pre-requisite for studying CSB effects. The complete LO calculation gives [51]

$$
A_{\mathrm{fb}}^{\mathrm{LO}}=(11.5 \pm 3.5) \times 10^{-4} \frac{\delta m_{N}^{\mathrm{str}}}{\mathrm{MeV}}
$$

which agrees nicely with the experimental data if one uses the value of $\delta m_{N}^{\text {str }}$ from Ref. [47]. We may also use (Eq. 4.1) to extract $\delta m_{N}^{\text {str }}$ from the above expression using the data, which yields

$$
\left.\delta m_{N}^{\text {str }}=(1.5 \pm 0.8 \text { (exp.) } \pm 0.5 \text { (th. })\right) \mathrm{MeV} .
$$

This result reveals a very good agreement with the one based on the Cottingham sum rule [47], $\delta m_{N}^{\text {str }}=2.0 \pm 0.3 \mathrm{MeV}$, and with a recent determination of the same quantity using lattice QCD [52], $\delta m_{N}^{\text {str }}=2.26 \pm 0.57 \pm 0.42 \pm 0.10 \mathrm{MeV}$.

\section{Summary}

We surveyed the recent developments for $N N \rightarrow N N \pi$. We showed, in particular, (see Ref. $[14,16])$ that the s-wave pion production amplitudes calculated up to NLO for $p p \rightarrow d \pi^{+}$provide a good qualitative understanding of the pion dynamics. However, the relatively large theoretical uncertainty of about $2 M_{\pi} / m_{N} \approx 30 \%$ for the cross section requires a computation of loops at NNLO. The latter are also absolutely necessary for $p p \rightarrow p p \pi^{0}$, see Ref. [53] for the first results in this direction. Recently, we have studied $p$-wave pion production up to NNLO [32]. In particular, we showed that it is possible to describe simultaneously the $p$-wave amplitudes in the $p n \rightarrow p p \pi^{-}$, $p p \rightarrow p n \pi^{+}, p p \rightarrow d \pi^{+}$channels by adjusting a single low-energy constant accompanying the short-range $(\bar{N} N)^{2} \pi$ operator available at NNLO. We also demonstrated that the problem with the simultaneous description of the weak proton-proton fusion process and $p p \rightarrow p n \pi^{+}$, reported in Ref. [31], is most probably due to the drawbacks of the partial wave analysis of Ref. [42] used in Ref. [31]. Based on good understanding of the pion production mechanisms in the isospin conserving case we studied charge symmetry breaking effects in $p n \rightarrow d \pi^{0}$. We performed a complete 
calculation of CSB effects at $\mathrm{LO}$ and extracted the strong contribution to the neutron-proton mass difference from this analysis. The value obtained, $\delta m_{N}^{\text {str }}=(1.5 \pm 0.8$ (exp.) \pm 0.5 (th.)) $\mathrm{MeV}$, is consistent with the result based on the Cottingham sum rule and with the recent lattice calculations. At present the uncertainty in this results is dominated by the experimental error bars - an improvement on this side would be very important. On the other hand, a calculation of higher order effects is also called for to confirm the theoretical uncertainty estimate.

\section{Acknowledgments}

I would like to thank E. Epelbaum, A. Filin, J. Haidenbauer, C. Hanhart, A. Kudryavtsev, V. Lensky and U.-G. Meißner for fruitful and enjoyable collaboration. I thank the organizers for the well-organized conference and for the invitation to give this talk. Work supported in parts by funds provided from the Helmholtz Association (grants VH-NG-222, VH-VI-231) and by the DFG (SFB/TR 16 and DFG-RFBR grant 436 RUS 113/991/0-1) and the EU HadronPhysics2 project. I acknowledge the support of the Federal Agency of Atomic Research of the Russian Federation.

\section{References}

[1] S. Weinberg, Physica A 96 (1979) 327.

[2] J. Gasser and H. Leutwyler. Ann. Phys. 158 (1984) 142.

[3] G. Colangelo, J. Gasser and H. Leutwyler, Nucl. Phys. B 603 (2001) 125 [arXiv:hep-ph/0103088].

[4] V. Bernard and U.-G. Meißner, Ann. Rev. Nucl. Part. Sci. 57 (2007) 33 [arXiv:hep-ph/0611231].

[5] P. F. Bedaque and U. van Kolck, Ann. Rev. Nucl. Part. Sci. 52 (2002) 339; [arXiv:nucl-th/0203055];

E. Epelbaum, Prog. Part. Nucl. Phys. 57 (2006) 654; [arXiv:nucl-th/0509032]; E. Epelbaum,

H.-W. Hammer and U.-G. Meißner, arXiv:0811.1338 [nucl-th], Rev. Mod. Phys., in print.

[6] T.D. Cohen et al., Phys. Rev. C 53 (1996) 2661 [arXiv:nucl-th/9512036].

[7] C. da Rocha, G. Miller and U. van Kolck, Phys. Rev. C 61 (2000) 034613 [arXiv:nucl-th/9904031].

[8] C. Hanhart, U. van Kolck, and G.A. Miller, Phys. Rev. Lett. 85 (2000) 2905 [arXiv:nucl-th/0004033].

[9] C. Hanhart and N. Kaiser, Phys. Rev. C 66 (2002) 054005 [arXiv:nucl-th/0208050].

[10] C. Hanhart, Phys. Rept. 397 (2004) 155 [arXiv:hep-ph/0311341].

[11] U. van Kolck, J. A. Niskanen and G. A. Miller, Phys. Lett. B 493 (2000) 65 [arXiv:nucl-th/0006042].

[12] A. K. Opper et al., Phys. Rev. Lett. 91 (2003) 212302 [arXiv:nucl-ex/0306027].

[13] S. Weinberg, Phys. Lett. B 295, 114 (1992).

[14] V. Lensky, et al., Eur. Phys. J. A 27 (2006) 37, [arXiv:nucl-th/0511054].

[15] A. Gårdestig, D. R. Phillips and C. Elster, Phys. Rev. C 73, 024002 (2006) [arXiv:nucl-th/0511042].

[16] V. Baru et al., Proceedings of MENU 2007, Julich, Germany, 10-14 Sep 2007, pp 128 [arXiv:0711.2748 [nucl-th]].

[17] D. A. Hutcheon et al., Nucl. Phys. A 535, 618 (1991).

[18] P. Heimberg et al., Phys. Rev. Lett. 77, 1012 (1996).

[19] M. Drochner et al., Nucl. Phys. A 643, 55 (1998). 
[20] P. Hauser et al., Phys. Rev. C 58, 1869 (1998).

[21] Th. Strauch et al., In Proceedings of EXA08, September 2008, Vienna, Austria, published in Hyperfine Interactions; Th. Strauch, PhD thesis, Cologne, 2009.

[22] D. Koltun and A. Reitan, Phys. Rev. 141, 1413 (1966).

[23] E. Epelbaum et al., Phys. Rev. C 66 (2002) 064001 [arXiv:nucl-th/0208023].

[24] V. Lensky et al., Eur. Phys. J. A 26 (2005) 107 [arXiv:nucl-th/0505039].

[25] V. Lensky et al., Eur. Phys. J. A 33 (2007) 339 [arXiv:0704.0443 [nucl-th]].

[26] A. Gårdestig and D. R. Phillips, Phys. Rev. C 73 (2006) 014002 [arXiv:nucl-th/0501049].

[27] A. Gårdestig, Phys. Rev. C 74 (2006) 017001 [arXiv:nucl-th/0604035].

[28] T. S. Park et al., Phys. Rev. C 67 (2003) 055206.

[29] A. Gårdestig and D. R. Phillips, Phys. Rev. Lett. 96 (2006) 232301.

[30] D. Gazit, S. Quaglioni and P. Navratil, P.R.L. 103, 102502 (2009) [arXiv:0812.4444 [nucl-th]].

[31] S. X. Nakamura, Phys. Rev. C 77 (2008) 054001.

[32] V. Baru et al, arXiv:0907.3911 [nucl-th], accepted for publication in Phys. Rev. C.

[33] B. G. Ritchie et al., Phys. Rev. C 47 (1993) 21.

[34] P. Heimberg et al., Phys. Rev. Lett. 77 (1996) 1012.

[35] M. Drochner et al. [GEM Collaboration], Nucl. Phys. A 643 (1998) 55.

[36] E. Korkmaz et al., Nucl. Phys. A 535 (1991) 637.

[37] E. L. Mathie et al., Nucl. Phys. A 397 (1983) 469.

[38] H. Hahn et al., Phys. Rev. Lett. 82 (1999) 2258.

[39] F. Duncan et al., Phys. Rev. Lett. 80 (1998) 4390.

[40] M. Daum et al., Eur. Phys. J. C 25 (2002) 55.

[41] A. Kacharava et al., "Spin physics from COSY to FAIR," arXiv:nucl-ex/0511028.

[42] R.W. Flammang et al., Phys. Rev. C 58 (1998) 916.

[43] R. Bilger et al., Nucl. Phys. A 693 (2001) 633.

[44] H.O. Meyer et al., Phys. Rev. Lett. 83 (1999) 5439; Phys. Rev. C 63 (2001) 064002.

[45] P. N. Deepak, J. Haidenbauer and C. Hanhart, Phys. Rev. C 72 (2005) 024004.

[46] E. J. Stephenson et al., Phys. Rev. Lett. 91 (2003) 142302.

[47] J. Gasser and H. Leutwyler, Phys. Rept. 87 (1982) 77.

[48] U.-G. Meißner and S. Steininger, Phys. Lett. B 419 (1998) 403.

[49] N. Fettes, U.-G. Meißner and S. Steininger, Phys. Lett. B 451 (1999) 233; N. Fettes and U.-G. Meißner, Phys. Rev. C 63 (2001) 045201; N. Fettes and U.-G. Meißner, Nucl. Phys. A 693 (2001) 693; M. Hoferichter, B. Kubis and U.-G. Meißner, Phys. Lett. B 678 (2009) 65.

[50] D. R. Bolton and G. A. Miller, arXiv:0907.0254 [nucl-th].

[51] A. Filin et al., arXiv:0907.4671 [nucl-th].

[52] S. R. Beane, K. Orginos and M. J. Savage, Nucl. Phys. B 768 (2007) 38.

[53] Y. Kim et al., Phys. Rev. C 80, 015206 (2009) [arXiv:0810.2774 [nucl-th]]. 